\title{
Near Optimal Solution for the Step Fixed Charge Transportation Problem
}

\author{
Khalid M. Altassan ${ }^{1}$, Mahmoud M. El-Sherbiny*2 ${ }^{2}$ and Bokkasam Sasidhar ${ }^{3}$ \\ ${ }^{1}$ King Saud University, Faculty of Business Administration, Saudi Arabia \\ ${ }^{2}$ Cairo University, Institute of Statistical Studies Research (ISSR), Operations Research Dept. Egypt \\ ${ }^{3}$ King Saud University, Faculty of Business Administration, Saudi Arabia
}

Received: 7 Oct. 2012, Revised: 22 Dec. 2012, Accepted: 25 Dec. 2012

Published online: 1 Jun. 2013

\begin{abstract}
Step Fixed-charge transportation problem (SFCTP) is considered to be one of the versions of Fixed-charge transportation problem (FCTP) where the fixed cost is incurred for every route that is used in the solution. This is considered to be an NP-hard problem since the cost structure causes the value of the objective function to behave like a step function. In this paper three formulae are proposed to construct intermediate coefficient matrix as a base for finding an initial solution for SFCTP. The proposed formulae overcome the drawbacks of one of the earlier proposed formulae, which fails to address the cases when load units become equal to or greater than the minimum of the supplies and demand for particular route. In addition, the achieved initial solution for the SFCTP is considered to be the best as compared to the initial solution achieved by the earlier proposed formulae in the literature. In order to confirm the superiority of the proposed formulae, forty problems with different sizes have been solved to evaluate and demonstrate the performance of the proposed formulae and to compare their performance with the earlier proposed formulae.
\end{abstract}

Keywords: Fixed charge transportation, Step fixed charge transportation, Heuristic algorithm.

\section{Introduction}

One of the versions of fixed charge transportation problem (FCTP) is the Step Fixed-charge transportation problem (SFCTP) where the fixed cost is incurred for every route that is used in the solution. In SFCTP, the fixed cost is proportional to the amount shipped. This cost structure causes the value of the objective function to behave like a step function. Unfortunately, not much research has been carried out in this area.

The FCTP is considered to be an NP-hard problem. After the fixed-charge problem was first formulated by Hirsch \& Dantzig in 1954 [1]. During 1961 Balinski [2] showed that fixed-charge transportation problem is a special case of fixed-charge problem and an approximate solution was presented. Since then, considerable research has been carried out on this topic. In 1988 Sandrock [3] analyzed the source induced fixed-charge transportation problem. FCTP is generally formulated and solved as a mixed integer network programming problem. Theoretically, the FCTP can be solved by using any mixed integer programming solving technique. However, these methods are not employed because of their inefficient and expensive computation.

Most of the solution methods of FCTP can be considered as either exact or heuristic. Exact methods include the cutting planes method [4], the vertex ranking method [5], and the branch-and-bound method [6] amongst others. These methods are generally not very useful when a problem reaches a certain level, because they do not make the most use of the special network structure of the FCTP. Therefore, heuristic methods have been proposed, such as the adjacent extreme point search method [2,7], the Lagrangian relaxation method [8,9] and such other heuristic methods $[10,11,12,13]$. Although these methods are usually computationally efficient, the major disadvantage of heuristic methods is the possibility of terminating at a local optimum that is far distant from the global optimum.

Heuristic techniques for solving FCTP have been proposed by Balinski [2]. These techniques start with constructing a coefficient matrix and finding the optimal solution based on it. After that Kowalski \& Lev [14] considered two more formulae in addition to Balinski's

\footnotetext{
*Corresponding author e-mail: msherbiny@cu.edu.eg
} 
and suggested a heuristic approach for improving the derived local optimal solution found based on the coefficient matrices which were arrived at using those formulae.

In order to improve the solution quality of the SFCTP, this paper critically analyses the erstwhile proposed heuristic formulae by Balinski [2] and Kowalski \& Lev [14] for constructing the coefficient matrix as a base for finding a good initial solution for SFCTPs. Further, three superior formulae have been proposed, which will also overcome the drawbacks of one of the earlier proposed formulae. In addition to that, forty problems with different dimensions have been solved to evaluate and demonstrate the performance of the proposed formulae and to compare their performance with the earlier proposed ones.

The rest of the paper is organized as follows: in section 2, SFCTP is described and its mathematical model is presented. The proposed formulae are described in section 3 , followed by two illustrative examples in section 4 . In section 5 the parametric analysis is carried out. Finally, the section 6 presents the conclusion and scope for future work.

\section{Description and modeling of SFCTP}

In this section the description and mathematical model of FCTP together with the modifications required to formulate SFCTP are presented.

The FCTP can be described as a distribution problem in which there are $m$ suppliers and $n$ customers. The suppliers denote warehouses, plants or factories while customers denote destinations or any demand points. Each of the $m$ suppliers can ship to any of the $n$ customers at a shipping cost per unit $c_{i j}$ (unit cost for shipping from supplier $i$ to customer $j$ ) plus a fixed cost $f_{i j}$, assumed for opening this route. Each supplier $i=1,2, \ldots, m$ has $s_{i}$ units of supply and each customer $j=1,2, \ldots, n$ demands $d_{j}$ units. $x_{i j}$ is the unknown quantity to be transported on the route $(i, j)$ from plant $i$ to customer $j$. The objective is to determine which routes are to be opened and the size of the shipment, so that the total cost of meeting demand, given the supply constraints, is minimized. The mathematical model of FCTP can be represented as in (1) to (4).

$$
\begin{gathered}
\text { Min } z=\sum_{i=1}^{m} \sum_{j=1}^{n}\left(c_{i j} x_{i j}+f_{i j} y_{i j}\right) \\
\text { s.t } \quad \sum_{i=1}^{m} x_{i j}=d_{j} \quad \text { for } j=1, \ldots, n \\
\sum_{j=1}^{n} x_{i j}=s_{i} \quad \text { for } i=1, \ldots, m \\
x_{i j} \geq 0 \quad \forall i, j \\
y_{i j}=0 \quad \text { if } \quad x_{i j}=0 \\
y_{i j}=1 \quad \text { if } \quad x_{i j}>0 \\
\text { and } \quad \sum_{i=1}^{m} s_{i}=\sum_{j=1}^{n} d_{j}
\end{gathered}
$$

In the SFCTP the fixed cost $f_{i j}$ for route $(i, j)$ is proportional to the transported amount through this route. So, an additional cost is added when the transported units exceeds a certain amount $A_{i j}$. The fixed cost $f_{i j}$ can be divided into two parts. The first part is $f_{i j, 1}$ which is the fixed cost to open a route $(i, j)$ as long as the transported quantity $x_{i j}$ is less than or equal to a certain amount $A_{i j}$. The second part $f_{i j, 2}$ which is the additional fixed cost applied when the transported quantity $x_{i j}$ exceeds this amount $A_{i j}$. Therefore the fixed cost $f_{i j}$ can be calculated by (5).

$$
f_{i j}=y_{i j, 1} f_{i j, 1}+y_{i j, 2} f_{i j, 2}
$$

where

$$
\begin{aligned}
& y_{i j, 1}=\left\{\begin{array}{l}
1 \text { if } x_{i j}>0 \\
0 \text { otherwise }
\end{array}\right. \\
& y_{i j, 2}=\left\{\begin{array}{l}
1 \text { if } x_{i j}>A_{i j} \\
0 \text { otherwise }
\end{array}\right.
\end{aligned}
$$

Incorporating (7) in the FCTP mathematical model, the standard mathematical model of the SFCTP can be represented as follows:

$$
\begin{aligned}
& \operatorname{Min} \quad z=\sum_{i=1}^{m} \sum_{j=1}^{n}\left(c_{i j} x_{i j}+y_{i j, 1} f_{i j, 1}+y_{i j, 2} f_{i j, 2}\right) \\
& \text { s.t } \quad \sum_{i=1}^{m} x_{i j}=d_{j} \quad \text { for } j=1, \ldots, n \\
& \sum_{j=1}^{n} x_{i j}=s_{i} \quad \text { fori }=1, \ldots, m \\
& x_{i j} \geq 0 \quad \forall i, j \\
& y_{i j}=0 \quad \text { if } \quad x_{i j}=0 \\
& y_{i j}=1 \quad \text { if } \quad x_{i j}>0 \\
& y_{i j, 1}=\left\{\begin{array}{l}
1 \text { if } x_{i j}>0 \\
0 \text { otherwise }
\end{array}\right. \\
& y_{i j, 2}=\left\{\begin{array}{l}
1 \text { if } x_{i j}>A_{i j} \\
0 \text { otherwise }
\end{array}\right.
\end{aligned}
$$

and $\quad \sum_{i=1}^{m} s_{i}=\sum_{j=1}^{n} d_{j}$ 
It can be observed that, if the shipment values $A_{i j}$ is greater than or equal to the $\operatorname{Min}\left(s_{i}, d_{j}\right) \forall i, j$ the optimal solution of SFCTP is an optimal solution of FCTP. i.e. If the $A_{i j} \geq \operatorname{Min}\left(s_{i}, d_{j}\right) \forall i, j$, the optimal solution of SFCTP is an optimal solution of FCTP. This solution will be the lower bound of all solutions of SFCTP.

\section{The Proposed Formula}

Balinski [2] has provided a heuristic solution for FCTP. Assuming the fixed cost as $f_{i j, 1}$ the Balinski matrix is obtained by formulating a linear version of FCTP by relaxing the integer restriction on $y_{i j}$ in (1)as follows, where $M_{i j}=\operatorname{Min}\left(s_{i}, d_{j}\right)$ :

$$
y_{i j}=x_{i j} / M_{i j}
$$

The linear version of FCTP will have the unit transportation cost of shipping through the route $(i, j)$ as follows:

$$
C_{i j}=f_{i j, 1} / M_{i j}+c_{i j}
$$

Since there is no algorithm for SFCTP, any heuristic method which provides a good solution is considered useful. In this direction Kowalski \& Lev [14] have put in efforts to propose two heuristic algorithms. In both the algorithms, the objective was to get a "good initial solution" and using this perturbing each load using single stepping-stone moves. In the first algorithm, the integer restriction considered in (9) by Balinski [2] has been replaced by $C_{i j}$ as represented in (10).

$$
C_{i j}=\left(f_{i j, 1+} f_{i j, 2}\right) / M_{i j}+c_{i j}
$$

In the second formula, the integer restriction considered in (9) by Balinski has been replaced by $C_{i j}$ as represented in (11).

$$
C_{i j}=f_{i j, 2} /\left(M_{i j}-A_{i j}\right)+c_{i j}
$$

A critical look at (11) reveals that the formulation fails to consider the cases when $A_{i j}=M_{i j}$ and $A_{i j}>M_{i j}$ as the values will be infinity when $A_{i j}=M_{i j}$ and assumes negative value in case $A_{i j}>M_{i j}$.

As illustrated in Fig. 1, [14] in the case of FCTP, for every loaded route $(i, j)$ the cost of the fixed-charge step function formulation is greater than the corresponding cost of the relaxed integer restriction function. The situation in case of SFCTP is illustrated in Fig. 2 [14]. The total cost $\left(T C_{i j}\right)$ for shipping $M_{i j}$ units through route $(i, j)$ can be calculated as represented in (12).

$$
T C_{i j}=f_{i j, 1}+c_{i j} A_{i j, 1}+f_{i j, 2}+c_{i j}\left(M_{i j}-A_{i j}\right)
$$

Further, (12) can be represented as in (13)

$$
T C_{i j}=f_{i j, 1}+f_{i j, 2}+c_{i j} M_{i j}
$$

The cost per unit $\left(C_{i j}\right)$ can be calculated by dividing (13) by $M_{i j}$ and can be representedas in (14).

$$
C_{i j}=\left(f_{i j, 1}+f_{i j, 2}\right) / M_{i j}+c_{i j}
$$

There are two cases of the shipped quantities which are $A_{i j}$ ? $M_{i j}$ and $A_{i j}<M_{i j}$. Therefore (14) can be represented as in (15).

$$
C_{i j}=\left\{\begin{array}{cc}
f_{i j, 1} / M_{i j}+c_{i j} & \text { if } A_{i j} \geq M_{i j} \\
\left(f_{i j, 1}+f_{i j, 2}\right) / M_{i j}+c_{i j} & \text { if } A_{i j}<M_{i j}
\end{array} \quad \forall i, j\right.
$$

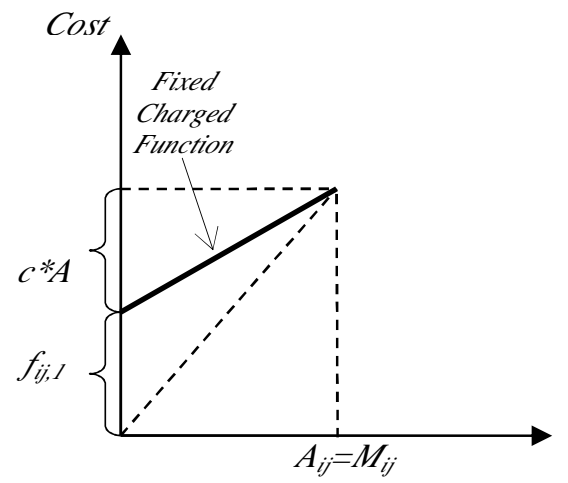

Quantity shipped

Fig. 1. Shipping costs as function of quantity shipped along route $(i, j)$ for FCTP

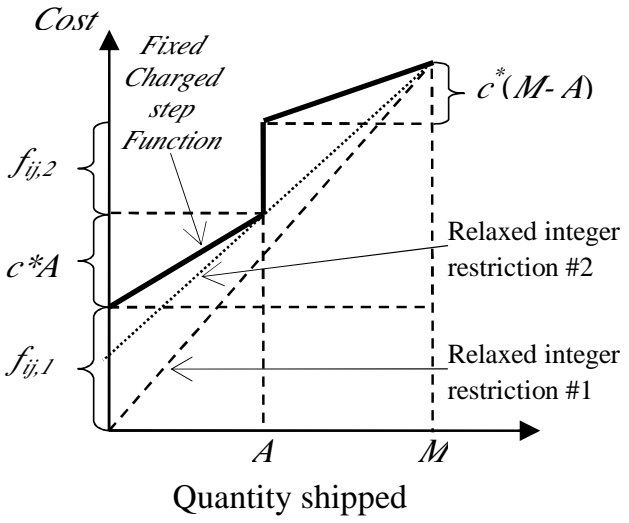

Fig. 2. Shipping costs as function of quantity shipped along route $(i, j)$ for SFCTP

Alternately, by considering that only $\left(M_{i j}-A_{i j}\right)$ units will be shipped through route $(i, j),(14)$ can be represented as (16).

$$
C_{i j}=\left\{\begin{array}{cc}
f_{i j, 1} / M_{i j}+c_{i j} & \text { if } A_{i j} \geq M_{i j} \\
f_{i j, 2} /\left(M_{i j}-A_{i j}\right)+c_{i j} & \text { if } A_{i j}<M_{i j}
\end{array} \quad \forall i, j\right.
$$


In the situation when only $\left(M_{i j}-A_{i j}\right)$ units will be shipped through route $(i, j)$, and another $\left(M_{i j}-A_{i j}\right)$ units shipped through another route, and if we consider that the cost of shipping all units as the cost through the route $(i$, $j$ ); the unit cost of this route can be represented as (17).

$$
C_{i j}=\left\{\begin{array}{cr}
f_{i j, 1} / M_{i j}+c_{i j} & \text { if } A_{i j} \geq M_{i j} \\
f_{i j, 2} / A_{i j}+f_{i j, 1} /\left(M_{i j}-A_{i j}\right)+c_{i j} & \text { if } A_{i j}<M_{i j}
\end{array}\right.
$$

This paper aims to propose three formulae as in (15), (16), and (17) for constructing intermediate coefficient matrix as a base for finding a local solution for SFCTP. Further a comparison of the performances and quality of these proposed formulae is undertaken with the earlier proposed formula (9) proposed by Balinski [2] and also with the two formulae (10) and (11) proposed by Kowalski \& Lev [14]. However, it has been pointed out that (11) fails to consider the cases when $A_{i j}=M_{i j}$ and $A_{i j}$ $>M_{i j}$ and hence the performance and quality comparison is restricted against (10) only.

\section{Illustrative Examples}

This section represents two illustrative examples used to compare the proposed three formulae (15), (16), and (17) with the previously proposed two (9) and (10).

In the first illustration, a $4 \times 5$ step transportation problem has been considered with parameters, viz., supplies $s_{i}$, demand $d_{j}$, variable costs $f_{i j, 1}$, fixed costs $f_{i j, 2}$, and step values $A_{i j}$ as in Table 1 .

The coefficient matrix generated using the formula (9) is shown in Table 2. The corresponding solution using QM for Windows Version 2.1 is presented in Table 3. The total fixed costs $f_{i j, 1}$ and $f_{i j, 2}$ are 150 and 200 respectively, the total variable cost $\sum_{i=1}^{m} \sum_{j=1}^{n} c_{i j} x_{i j}$ is 580, and the total cost is 930 .

Table 1: The parameters and variables of example $4 \times 5$

\begin{tabular}{|c|c|c|c|c|c|c|c|c|c|c|}
\hline & 1 & 2 & 3 & 4 & 5 & 1 & 2 & 3 & 4 & 5 \\
\hline & \multicolumn{10}{|l|}{$d_{j}$} \\
\hline & 40 & 20 & 70 & 10 & 60 & & & & & \\
\hline$s_{i}$ & \multicolumn{5}{|c|}{ Variable cost $c_{i j}$} & \multicolumn{5}{|c|}{ Fixed $\operatorname{cost} f_{i j, 1}$} \\
\hline 10 & 5 & 3 & 2 & 4 & 6 & 40 & 20 & 30 & 20 & 10 \\
\hline 100 & 3 & 5 & 3 & 4 & 3 & 10 & 20 & 20 & 30 & 20 \\
\hline 20 & 3 & 4 & 6 & 5 & 2 & 40 & 30 & 10 & 20 & 30 \\
\hline \multirow[t]{6}{*}{70} & 2 & 5 & 4 & 3 & 4 & 10 & 40 & 40 & 10 & 10 \\
\hline & \multicolumn{5}{|c|}{ Fixed cost $f_{i j, 2}$} & \multicolumn{5}{|c|}{ Step value $A_{i j}$} \\
\hline & 50 & 70 & 80 & 70 & 80 & 20 & 20 & 20 & 20 & 20 \\
\hline & 60 & 70 & 60 & 80 & 60 & 20 & 20 & 20 & 20 & 20 \\
\hline & 60 & 80 & 80 & 70 & 70 & 20 & 20 & 20 & 20 & 20 \\
\hline & 80 & 40 & 50 & 50 & 50 & 20 & 20 & 20 & 20 & 20 \\
\hline
\end{tabular}

Table 2: The coefficient matrix using formula (9)

\begin{tabular}{l|lllll}
\hline & $d_{1}$ & $d_{2}$ & $d_{3}$ & $d_{4}$ & $d_{5}$ \\
\hline$s_{1}$ & 9.0 & 5.0 & 5.0 & 6.0 & 7.0 \\
$s_{2}$ & 3.3 & 6.0 & 3.3 & 7.0 & 3.3 \\
$s_{3}$ & 5.0 & 5.5 & 6.5 & 7.0 & 3.5 \\
$s_{4}$ & 2.3 & 7.0 & 4.6 & 4.0 & 4.2 \\
\hline
\end{tabular}

$\forall i, j$ Thle 3: Optimal distribution for formula

\begin{tabular}{l|lllll} 
& $d_{1}$ & $d_{2}$ & $d_{3}$ & $d_{4}$ & $d_{5}$ \\
\hline$s_{1}$ & & 10 & & & \\
$s_{2}$ & & & 70 & & 30 \\
$s_{3}$ & & 10 & & & 10 \\
$s_{4}$ & 40 & & & 10 & 20 \\
\hline
\end{tabular}

Similarly, the coefficient matrix generated using the formula (10) is shown in Table 4. The corresponding solution is presented in Table 5. The total fixed costs $f_{i j, 1}$ and $f_{i j, 2}$ are 110 and 250 respectively, the total variable cost is 620, and the total cost is 980 .

Table 4: The coefficient matrix using formula

\begin{tabular}{l|lllll}
\hline & $d_{1}$ & $d_{2}$ & $d_{3}$ & $d_{4}$ & $d_{5}$ \\
\hline$s_{1}$ & 14.0 & 12.0 & 13.0 & 13.0 & 15.0 \\
$s_{2}$ & 4.8 & 9.5 & 4.1 & 15.0 & 4.3 \\
$s_{3}$ & 8.0 & 9.5 & 10.5 & 14.0 & 7.0 \\
$s_{4}$ & 4.3 & 9.0 & 5.3 & 9.0 & 5.0 \\
\hline
\end{tabular}

The coefficient matrix generated using the formulae (15) and (16) are shown in Tables 6 and 7 respectively. It is observed that using (15) and (16), we obtain the same local optimal solution, as presented in Table 8. The total fixed costs $f_{i j, 1}$ and $f_{i j, 2}$ are 140 and 140 respectively, the total variable cost is 580 , and the total cost is 860 .

Table 5: Optimal distribution for formula (10)

\begin{tabular}{l|lllll}
\hline & $d_{1}$ & $d_{2}$ & $d_{3}$ & $d_{4}$ & $d_{5}$ \\
\hline$s_{1}$ & & 0 & & 10 & \\
$s_{2}$ & & & 70 & & 30 \\
$s_{3}$ & & 20 & & & 0 \\
$s_{4}$ & 40 & & & & 30 \\
\hline
\end{tabular}

Table 6: The coefficient matrix using formula (15)

\begin{tabular}{l|lllll}
\hline & $d_{1}$ & $d_{2}$ & $d_{3}$ & $d_{4}$ & $d_{5}$ \\
\hline$s_{1}$ & 9.0 & 5.0 & 5.0 & 6.0 & 7.0 \\
$s_{2}$ & 4.8 & 6.0 & 4.1 & 7.0 & 4.3 \\
$s_{3}$ & 5.0 & 5.5 & 6.5 & 7.0 & 3.5 \\
$s_{4}$ & 4.3 & 7.0 & 5.3 & 4.0 & 5.0 \\
\hline
\end{tabular}

Table 7: The coefficient matrix using formula (16)

\begin{tabular}{l|lllll}
\hline & $d_{1}$ & $d_{2}$ & $d_{3}$ & $d_{4}$ & $d_{5}$ \\
\hline$s_{1}$ & 9.0 & 5.0 & 5.0 & 6.0 & 7.0 \\
$s_{2}$ & 6.0 & 6.0 & 4.2 & 7.0 & 4.5 \\
$s_{3}$ & 5.0 & 5.5 & 6.5 & 7.0 & 3.5 \\
$s_{4}$ & 6.0 & 7.0 & 5.0 & 4.0 & 5.3 \\
\hline
\end{tabular}


Table 8: Optimal distribution for formula (15) and (16)

\begin{tabular}{l|lllll}
\hline & $d_{1}$ & $d_{2}$ & $d_{3}$ & $d_{4}$ & $d_{5}$ \\
\hline$s_{1}$ & & 10 & & & \\
$s_{2}$ & & 10 & 70 & & 20 \\
$s_{3}$ & & & & & 20 \\
$s_{4}$ & 40 & & & 10 & 20 \\
\hline
\end{tabular}

The coefficient matrix generated using the formula (17) is shown in Table 9. The corresponding solution is presented in Table 10. The total fixed costs $f_{i j, 1}$ and $f_{i j, 2}$ are 150 and 140 respectively, the total variable cost is 590 , and the total cost is 880 .

Table 9: The coefficient matrix using formula (17)

\begin{tabular}{l|lllll}
\hline & $d_{1}$ & $d_{2}$ & $d_{3}$ & $d_{4}$ & $d_{5}$ \\
\hline$s_{1}$ & 9.0 & 5.0 & 5.0 & 6.0 & 7.0 \\
$s_{2}$ & 6.5 & 6.0 & 6.4 & 7.0 & 6.5 \\
$s_{3}$ & 5.0 & 5.5 & 6.5 & 7.0 & 3.5 \\
$s_{4}$ & 6.5 & 7.0 & 7.3 & 4.0 & 6.8 \\
\hline
\end{tabular}

Table 10: Optimal distribution for formula

\begin{tabular}{l|lllll}
\hline & $d_{1}$ & $d_{2}$ & $d_{3}$ & $d_{4}$ & $d_{5}$ \\
\hline$s_{1}$ & & & 10 & & \\
$s_{2}$ & & 20 & 60 & & 20 \\
$s_{3}$ & & & & & 20 \\
$s_{4}$ & 40 & & & 10 & 20 \\
\hline
\end{tabular}

The comparative statement of the total costs for the illustration using the different formulae is summarized in Table 11.

Table 11: Summary of total costs using different formulae.

\begin{tabular}{l|l|l|c|l}
\multicolumn{2}{l|}{$\begin{array}{l}\text { Formula } \\
\text { formulae. }\end{array}$} & $f_{i j, 1}$ & $\sum_{i=1}^{m} \sum_{j=1}^{n} c_{i j} x_{i j}$ & $\begin{array}{l}\text { Total } \\
\text { Cost }\end{array}$ \\
\hline$(10)$ & 150 & 200 & 580 & 930 \\
$(11)$ & 110 & 250 & 620 & 980 \\
$(14)$ & 140 & 140 & 580 & 860 \\
$(15)$ & 140 & 140 & 580 & 860 \\
$(16)$ & 150 & 140 & 590 & 880 \\
\hline
\end{tabular}

As summarized in Table 11, the results using the proposed three formulae 15,16 and 17 are superior to the ones proposed earlier. Further, the quality of the results using the formulae 14 and 15 are superior to the results obtained using 16.

In the next illustration, a problem with a higher dimension, viz., $5 \times 10$ has been considered. Table 12 gives the parameters and variables used for the example of size $5 \times 10$.
Table 12: The parameters and variables of example $5 \times 10$

\begin{tabular}{|c|c|c|c|c|c|c|c|c|c|c|}
\hline & 1 & 2 & 3 & 4 & 5 & 6 & 7 & 8 & 9 & 10 \\
\hline & \multicolumn{10}{|l|}{$d_{j}$} \\
\hline & 40 & 20 & 50 & 10 & 10 & 20 & 30 & 30 & 50 & 40 \\
\hline$s_{i}$ & \multicolumn{10}{|c|}{ Variable cost $c_{i j}$} \\
\hline 20 & 4 & 5 & 5 & 2 & 2 & 4 & 4 & 2 & 8 & 4 \\
\hline 40 & 4 & 4 & 7 & 5 & 6 & 5 & 7 & 6 & 7 & 5 \\
\hline 90 & 4 & 6 & 3 & 8 & 4 & 3 & 3 & 3 & 5 & 7 \\
\hline 60 & 5 & 6 & 3 & 6 & 6 & 4 & 6 & 8 & 2 & 2 \\
\hline \multirow[t]{19}{*}{90} & 3 & 5 & 5 & 8 & 3 & 8 & 5 & 7 & 4 & 6 \\
\hline & \multicolumn{10}{|c|}{ Fixed cost $f_{i j, 1}$} \\
\hline & 100 & 170 & 190 & 100 & 170 & 150 & 190 & 170 & 150 & 200 \\
\hline & 110 & 170 & 170 & 200 & 180 & 160 & 180 & 180 & 170 & 140 \\
\hline & 120 & 120 & 170 & 100 & 120 & 170 & 130 & 160 & 110 & 190 \\
\hline & 130 & 120 & 130 & 180 & 160 & 140 & 170 & 180 & 190 & 110 \\
\hline & 110 & 180 & 160 & 170 & 130 & 120 & 110 & 160 & 160 & 120 \\
\hline & \multicolumn{10}{|l|}{$\boldsymbol{A}_{i j}$} \\
\hline & 40 & 30 & 40 & 50 & 40 & 30 & 20 & 40 & 50 & 40 \\
\hline & 10 & 50 & 30 & 40 & 30 & 50 & 20 & 30 & 20 & 10 \\
\hline & 50 & 40 & 40 & 10 & 50 & 20 & 30 & 10 & 30 & 20 \\
\hline & 40 & 10 & 30 & 20 & 20 & 40 & 50 & 20 & 20 & 30 \\
\hline & 20 & 30 & 20 & 20 & 10 & 30 & 50 & 20 & 40 & 50 \\
\hline & \multicolumn{10}{|c|}{ Fixed $\operatorname{cost} f_{i j, 2}$} \\
\hline & 210 & 400 & 280 & 370 & 320 & 210 & 300 & 220 & 230 & 210 \\
\hline & 290 & 340 & 340 & 280 & 360 & 330 & 200 & 390 & 310 & 400 \\
\hline & 360 & 300 & 330 & 290 & 290 & 400 & 310 & 210 & 350 & 390 \\
\hline & 390 & 220 & 220 & 250 & 330 & 290 & 370 & 310 & 350 & 280 \\
\hline & 340 & 320 & 270 & 270 & 270 & 320 & 360 & 220 & 370 & 280 \\
\hline
\end{tabular}

The coefficient matrix and the corresponding solution generated using the formula (9) is shown in Tables 13 and 14. The total fixed costs $f_{i j, 1}$ and $f_{i j, 2}$ are 1790 and 1200 respectively, the total variable cost is 960 , and the total cost is 3950 .

Table 13: The coefficient matrix using formula (9)

\begin{tabular}{l|llllllllll}
\hline & $d_{1}$ & $d_{2}$ & $d_{3}$ & $d_{4}$ & $d_{5}$ & $d_{6}$ & $d_{7}$ & $d_{8}$ & $d_{9}$ & $d_{10}$ \\
\hline$s_{1}$ & 9.0 & 13.5 & 14.5 & 12.0 & 19.0 & 11.5 & 13.5 & 10.5 & 15.5 & 14.0 \\
$s_{2}$ & 6.8 & 12.5 & 11.3 & 25.0 & 24.0 & 13.0 & 13.0 & 12.0 & 11.3 & 8.5 \\
$s_{3}$ & 7.0 & 12.0 & 6.4 & 18.0 & 16.0 & 11.5 & 7.3 & 8.3 & 7.2 & 11.8 \\
$s_{4}$ & 8.3 & 12.0 & 5.6 & 24.0 & 22.0 & 11.0 & 11.7 & 14.0 & 5.8 & 4.8 \\
$s_{5}$ & 5.8 & 14.0 & 8.2 & 25.0 & 16.0 & 14.0 & 8.7 & 12.3 & 7.2 & 9.0 \\
\hline
\end{tabular}

Table 14: Optimal distribution for formula (9)

\begin{tabular}{l|cccccccccc}
\hline & $d_{1}$ & $d_{2}$ & $d_{3}$ & $d_{4}$ & $d_{5}$ & $d_{6}$ & $d_{7}$ & $d_{8}$ & $d_{9}$ & $d_{10}$ \\
\hline$s_{1}$ & & & & 10 & & 10 & & & & \\
$s_{2}$ & 10 & 20 & & & & 10 & & & & \\
$s_{3}$ & & & 30 & & & & 30 & 30 & & \\
$s_{4}$ & & & 20 & & & & & & 0 & 40 \\
$s_{5}$ & 30 & & & & 10 & & & & 50 & \\
\hline
\end{tabular}

Tables 15 and 16 give the coefficient matrix and the corresponding solution generated using the formula (10). The total fixed costs $f_{i j, 1}$ and $f_{i j, 2}$ are 1500 and 1640 respectively, the total variable cost is 1140 , and the total 
cost is 4280 .

Table 15: The coefficient matrix using formula (10) \begin{tabular}{l|llllllllll}
\hline & $d_{1}$ & $d_{2}$ & $d_{3}$ & $d_{4}$ & $d_{5}$ & $d_{6}$ & $d_{7}$ & $d_{8}$ & $d_{9}$ & $d_{10}$ \\
\hline$s_{1}$ & 19.5 & 33.5 & 28.5 & 49.0 & 51.0 & 22.0 & 28.5 & 21.5 & 27.0 & 24.5 \\
$s_{2}$ & 14.0 & 29.5 & 19.8 & 53.0 & 60.0 & 29.5 & 19.7 & 25.0 & 19.0 & 18.5 \\
$s_{3}$ & 16.0 & 27.0 & 13.0 & 47.0 & 45.0 & 31.5 & 17.7 & 15.3 & 14.2 & 21.5 \\
$s_{4}$ & 18.0 & 23.0 & 10.0 & 49.0 & 55.0 & 25.5 & 24.0 & 24.3 & 12.8 & 11.8 \\
$s_{5}$ & 14.3 & 30.0 & 13.6 & 52.0 & 43.0 & 30.0 & 20.7 & 19.7 & 14.6 & 16.0 \\
\hline
\end{tabular}

Tables 17 and 18 give the coefficient matrix and the corresponding solution generated using the formula (15). The total fixed costs $f_{i j, 1}$ and $f_{i j, 2}$ are 1770 and 220 respectively, the total variable cost is 1150 , and the total cost is 3140 .

Table 16: Optimal distribution for formula (10)

\begin{tabular}{l|llllllllll}
\hline & $d_{1}$ & $d_{2}$ & $d_{3}$ & $d_{4}$ & $d_{5}$ & $d_{6}$ & $d_{7}$ & $d_{8}$ & $d_{9}$ & $d_{10}$ \\
\hline$s_{1}$ & & & & 0 & & 20 & & & & \\
$s_{2}$ & 40 & & & & & & 0 & & & \\
$s_{3}$ & & & 20 & 10 & & & 30 & 30 & & \\
$s_{4}$ & & 20 & 0 & & & & & & & 40 \\
$s_{5}$ & & & 30 & & 10 & & & & 50 & \\
\hline
\end{tabular}

Table 17: The coefficient matrix using formula (15)

\begin{tabular}{l|llllllllll}
\hline & $d_{1}$ & $d_{2}$ & $d_{3}$ & $d_{4}$ & $d_{5}$ & $d_{6}$ & $d_{7}$ & $d_{8}$ & $d_{9}$ & $d_{10}$ \\
\hline$s_{1}$ & 9.0 & 13.5 & 14.5 & 12.0 & 19.0 & 11.5 & 13.5 & 10.5 & 15.5 & 14.0
\end{tabular}

\begin{tabular}{l|llllllllll}
$s_{2}$ & 14.0 & 12.5 & 19.8 & 25.0 & 24.0 & 13.0 & 19.7 & 12.0 & 19.0 & 18.5
\end{tabular}

\begin{tabular}{l|lllllllllll}
$s_{3}$ & 7.0 & 12.0 & 13.0 & 18.0 & 16.0 & 11.5 & 7.3 & 15.3 & 14.2 & 21.5
\end{tabular}

$\begin{array}{llllllllllll}s_{4} & 8.3 & 23.0 & 10.0 & 24.0 & 22.0 & 11.0 & 11.7 & 24.3 & 12.8 & 11.8\end{array}$

\begin{tabular}{l|llllllllll}
$s_{5}$ & 14.3 & 14.0 & 13.6 & 25.0 & 16.0 & 14.0 & 8.7 & 19.7 & 14.6 & 9.0
\end{tabular}

Table 18: Optimal distribution for formula (15)

\begin{tabular}{l|llllllllll}
\hline & $d_{1}$ & $d_{2}$ & $d_{3}$ & $d_{4}$ & $d_{5}$ & $d_{6}$ & $d_{7}$ & $d_{8}$ & $d_{9}$ & $d_{10}$ \\
\hline$s_{1}$ & & & & 10 & & 0 & & 10 & & \\
$s_{2}$ & & 20 & & & & & & 20 & & \\
$s_{3}$ & 40 & & & & & 20 & 30 & & & \\
$s_{4}$ & & & 50 & & & 0 & & & 10 & \\
$s_{5}$ & & & & & 10 & & & & 40 & 40 \\
\hline
\end{tabular}

Corresponding Tables using formula (16) are Tables 19 and 20 , resulting in the total fixed costs $f_{i j, 1}$ and $f_{i j, 2}$ as 1770 and 620 respectively, the total variable cost $\sum_{i=1}^{m} \sum_{j=1}^{n} c_{i j} x_{i j}$ is 1190 , and the total cost is 3580 .

Table 19: The coefficient matrix using formula (16)

\begin{tabular}{l|llllllllll}
\hline & $d_{1}$ & $d_{2}$ & $d_{3}$ & $d_{4}$ & $d_{5}$ & $d_{6}$ & $d_{7}$ & $d_{8}$ & $d_{9}$ & $d_{10}$ \\
\hline$s_{1}$ & 9.0 & 13.5 & 14.5 & 12.0 & 19.0 & 11.5 & 13.5 & 10.5 & 15.5 & 14.0 \\
$s_{2}$ & 13.7 & 12.5 & 41.0 & 25.0 & 24.0 & 13.0 & 27.0 & 12.0 & 22.5 & 18.3 \\
$s_{3}$ & 7.0 & 12.0 & 36.0 & 18.0 & 16.0 & 11.5 & 7.3 & 13.5 & 22.5 & 26.5 \\
$s_{4}$ & 8.3 & 28.0 & 14.0 & 24.0 & 22.0 & 11.0 & 11.7 & 39.0 & 13.7 & 30.0 \\
$s_{5}$ & 20.0 & 14.0 & 14.0 & 25.0 & 16.0 & 14.0 & 8.7 & 29.0 & 41.0 & 9.0 \\
\hline
\end{tabular}

Table 20: Optimal distribution for formula (16)

\begin{tabular}{l|llllllllll}
\hline & $d_{1}$ & $d_{2}$ & $d_{3}$ & $d_{4}$ & $d_{5}$ & $d_{6}$ & $d_{7}$ & $d_{8}$ & $d_{9}$ & $d_{10}$ \\
\hline$s_{1}$ & & & & 10 & & & & 10 & & \\
$s_{2}$ & & 20 & & & & & & 20 & & \\
$s_{3}$ & 40 & 0 & & & 10 & 10 & 30 & & & \\
$s_{4}$ & & & 0 & & & 10 & & & 50 & \\
$s_{5}$ & & & 50 & & & & & & & 40 \\
\hline
\end{tabular}

Corresponding Tables using formula (17) are Tables 21 and 22 , resulting in the total fixed costs $f_{i j, 1}$ and $f_{i j, 2}$ as 1810 and 220 respectively, the total variable cost $\sum_{i=1}^{m} \sum_{j=1}^{n} c_{i j} x_{i j}$ is 1460 , and the total cost is 3490 .

Table 21: The coefficient matrix using formula (17) \begin{tabular}{l|llllllllll}
\hline & $d_{1}$ & $d_{2}$ & $d_{3}$ & $d_{4}$ & $d_{5}$ & $d_{6}$ & $d_{7}$ & $d_{8}$ & $d_{9}$ & $d_{10}$ \\
\hline$s_{1}$ & 9.0 & 13.5 & 14.5 & 12.0 & 19.0 & 11.5 & 13.5 & 10.5 & 15.5 & 14.0 \\
$s_{2}$ & 36.7 & 12.5 & 35.3 & 25.0 & 24.0 & 13.0 & 35.0 & 12.0 & 31.0 & 49.7 \\
$s_{3}$ & 7.0 & 12.0 & 28.3 & 18.0 & 16.0 & 11.5 & 7.3 & 32.0 & 22.2 & 36.0 \\
$s_{4}$ & 8.3 & 40.0 & 16.8 & 24.0 & 22.0 & 11.0 & 11.7 & 41.5 & 25.8 & 22.3 \\
$s_{5}$ & 25.5 & 14.0 & 23.8 & 25.0 & 16.0 & 14.0 & 8.7 & 34.0 & 29.3 & 9.0 \\
\hline
\end{tabular}

Table 22: Optimal distribution for formula (17)

\begin{tabular}{l|llllllllll}
\hline & $d_{1}$ & $d_{2}$ & $d_{3}$ & $d_{4}$ & $d_{5}$ & $d_{6}$ & $d_{7}$ & $d_{8}$ & $d_{9}$ & $d_{10}$ \\
\hline$s_{1}$ & & & & & & & & & 20 & \\
$s_{2}$ & & 10 & & & & & & 30 & & \\
$s_{3}$ & 40 & 0 & & 10 & & 10 & & & 30 & \\
$s_{4}$ & & & 50 & & & 10 & & & & \\
$s_{5}$ & & 10 & & & 10 & & 30 & & & 40 \\
\hline
\end{tabular}

The comparative statement of the total costs for the illustration using the different formulae is summarized in Table 23.

Table 23: Summary of total costs using different formulae.

\begin{tabular}{l|c|l|c|c}
\hline Formula & $f_{i j, 1}$ & $f_{i j, 2}$ & $\sum_{i=1}^{m} \sum_{j=1}^{n} c_{i j} x_{i j}$ & $\begin{array}{c}\text { Total } \\
\text { Cost }\end{array}$ \\
\hline$(10)$ & 1790 & 1200 & 960 & 3950 \\
$(11)$ & 1500 & 1640 & 1140 & 4280 \\
$(14)$ & 1770 & 220 & 1150 & 3140 \\
$(15)$ & 1770 & 620 & 1190 & 3580 \\
$(16)$ & 1810 & 220 & 1460 & 3490 \\
\hline
\end{tabular}

As summarized in Table 23, the results using the proposed three formulae 15, 16 and 17 are superior to the ones proposed earlier. Further, the quality of the results using the formula 15 is superior to the results obtained using rest of the formulae.

From the above two illustrations it can be observed that there exist formulations of the intermediate coefficient matrix $C_{i j}$ which yield superior coefficient matrix as a base for finding a local solution for SFCTPs as compared to the earlier proposed formulations. In order to further explore the effectiveness of the proposed formulae, the results based on different problems with 
eight dimensions ranging from $3 \times 3$ to $20 \times 20$ and with different $A_{i j}$ were analyzed. The values of $A_{i j}$ were considered as both fixed as well as variable values for different problems. The details of analysis and the results are presented in the next section.

\section{Parametric analysis}

In this section different illustrative examples are considered to discover the best formulation of the function for determining the intermediate coefficient matrix, $C_{i j}$ from among the earlier proposed two and the newly proposed three formulations. Random numbers were generated using Excel for determining the problem parameters and for generating the coefficient matrix corresponding to each formulation. The problem is solved using the Transportation Module using QM for Windows, Version 2.1. These solutions are taken to Excel sheet to find the corresponding fixed costs, variable costs and total costs for each problem. Because the scale of the functions in each problem will be different, they cannot be compared directly. Therefore, the Relative Percentage Deviation (RPD) is used for each combinatioin [15]. RPD is calculated by using (18).

$$
R P D=\frac{A \lg _{\text {sol }}-\text { Min }_{\text {sol }}}{\text { Min }_{\text {sol }}} \times 100
$$

where $\mathrm{Alg}_{\text {sol }}$ and $\mathrm{Min}_{\text {sol }}$ are the obtained $T C_{i j}$ values for each replication of trial for a given dimension and the obtained best solution, respectively. After converting the objective values to $R P D \mathrm{~s}$, the mean $R P D$ is calculated for each dimension. Problems with eight dimensions ranging from $3 \times 3$ to $15 \times 20$ were considered for illustrations. For each dimension, five problems with different values for each characteristic (both fixed and variable values) have been generated and used to calculate the average costs and RPD values for each dimension. Thus, a sample of 40 problems have been generated and solved. The characteristics of the test problems considered are presented in Table 24.

Table 24: Characteristics of SFCT test problems

\begin{tabular}{lllllllll}
\hline $\begin{array}{l}\text { Pro. } \\
\text { size }\end{array}$ & $\begin{array}{l}\text { Range } \\
\text { of }\left(\mathrm{d}_{j}\right) \\
\left(\mathrm{s}_{i}\right)\end{array}$ & $\begin{array}{l}\text { Rang } \\
\text { of }\left(\mathrm{c}_{i j}\right)\end{array}$ & $\begin{array}{l}\text { Rang } \\
\text { of }\left(\mathrm{f}_{i j, 1}\right)\end{array}$ & $\begin{array}{l}\text { Rang } \\
\text { of }\left(\mathrm{f}_{i j, 2}\right)\end{array}$ \\
\hline \multicolumn{1}{c}{ LL } & UL & LL & UL & LL & UL & LL & UL \\
\hline $3 \times 3$ & 50 & 100 & 1 & 3 & 10 & 20 & 20 & 50 \\
$4 \times 5$ & 150 & 250 & 1 & 9 & 10 & 40 & 30 & 70 \\
$5 \times 10$ & 200 & 500 & 1 & 9 & 10 & 50 & 30 & 90 \\
$10 \times 10$ & 300 & 500 & 1 & 9 & 100 & 200 & 200 & 400 \\
$10 \times 15$ & 500 & 1000 & 1 & 9 & 100 & 500 & 200 & 600 \\
$15 \times 15$ & 500 & 2000 & 1 & 9 & 100 & 500 & 200 & 600 \\
$15 \times 20$ & 1000 & 3000 & 1 & 9 & 100 & 500 & 200 & 700 \\
$20 \times 20$ & 1000 & 3000 & 1 & 9 & 100 & 500 & 200 & 700 \\
\hline
\end{tabular}

All the 40 problems considered were solved to find the total cost of the associated SFCTP and subsequently the corresponding $R P D$ s for each of the earlier proposed two and the newly proposed three formulae. The values of average $R P D$ s, based on five illustrative examples for each of the eight dimensions considered using the five formulae and the overall mean $R P D$ for each of the formulae are presented in Table 25.

Based on the results presented in Table 25, the overall mean RPD of formula (15) is providing the least value as compared to the other formulae. This is followed by the other two proposed formulae, viz., (17) and (16) respectively. Hence, it can be concluded that the newly proposed three formulae are superior and can be used as a better alternative for constructing coefficient matrix as a base for finding a local solution for SFCTPs as compared to the earlier used formulae (9) and (10).

Table 25: The comparitive results of the averageRPD for the proposed formulae

\begin{tabular}{c|ccccccccc}
\hline $\begin{array}{l}\text { Form- } \\
\text { ulas }\end{array}$ & \multicolumn{1}{|c}{ Average RPD of the test problems } & \multicolumn{3}{c}{$\begin{array}{l}\text { Mean } \\
R P D\end{array}$} \\
\hline & $3 \times 3$ & $4 \times 5$ & $5 \times 10$ & $10 \times 1010 \times 1515 \times 1515 \times 2020 \times 20$ \\
\hline$(10)$ & 2.1 & 4.0 & 10.5 & 10.2 & 9.1 & 2.3 & 2.4 & 14.5 & 6.89 \\
$(11)$ & 4.6 & 3.4 & 12.4 & 8.6 & 8.7 & 6.6 & 1.3 & 16.2 & 7.73 \\
$(15)$ & 0.0 & 2.9 & 0.0 & 0.0 & 0.0 & 0.0 & 0.0 & 0.0 & 0.36 \\
$(16)$ & 0.5 & 3.4 & 7.3 & 11.0 & 8.1 & 0.5 & 2.9 & 7.7 & 5.18 \\
$(17)$ & 3.6 & 0.0 & 9.5 & 3.8 & 8.4 & 1.7 & 9.4 & 3.8 & 5.03 \\
\hline
\end{tabular}

In addition to the above, in order to statistically test the significance of effectiveness of the results using different formulae, paired sample t-tests were used to determine the significant differences in the $R P D$ values obtained using the five formulations, for each of the pairs. For the purpose of comparisons the $R P D$ values obtained using all the 40 problems were used. The results of the tests are summarized in Table 26.

Table 26: The p-values of paired sample t-tests

\begin{tabular}{l|llll}
\hline Formulae & \multicolumn{4}{|l}{-value(2-Tailed) } \\
\hline & $(17)$ & $(16)$ & $(15)$ & $(11)$ \\
\hline$(10)$ & 0.293 & 0.188 & 0.000 & 0.530 \\
$(11)$ & 0.155 & 0.107 & 0.000 & \\
$(15)$ & 0.000 & 0.000 & & \\
$(16)$ & 0.993 & & & \\
\hline
\end{tabular}

As illustrated in Table 26, it can be concluded at 0.01 level of significance the quality of the results using the proposed formula (15) provides the coefficient matrix which yields the total cost which is significantly lower than those provided by the rest of the formulae. Hence, the proposed formula (15) can be considered as the best alternative as compared to the formulae provided by 
Balinski [2] and Kowalski \& Lev [14] for solving SFCTPs.

\section{Conclusion}

Three formulae have been proposed in this paper for constructing intermediate coefficient matrix as a basis for finding a local solution for SFCTPs. In addition, a comparison of the performances and quality of these proposed formulae is undertaken with the earlier proposed formulae proposed by Balinski [2] and also with the two formulae proposed by Kowalski \& Lev [14]. It is proved that one of the formulae (11) proposed by Kowalski \& Lev [14] fails to consider the cases when $A_{i j}$ $=M_{i j}$ and $A_{i j}>M_{i j}$ as the values will be infinity when $A_{i j}$ $=M_{i j}$ and assumes negative value in case $A_{i j}>M_{i j}$. In order to compare the formulae for their effectiveness, the results based on different problems with differing dimensions and with different $\mathrm{A}_{i j}$ were analyzed. Tests of hypotheses were performed and proved that one of the proposed formulae (15) provides the intermediate coefficient matrix $C_{i j}$ which yields significantly lower total costs as compared to the remaining formulae.

Further work includes more experiments with the parameters of SFCTP and testing the proposed SFCTP on other real life problems. In addition, investigating the usage of metaheuristic techniques such as artificial immune systems, tabu search, particle awarm, simulated annealing and genetic algorithms for solving SFCTP will be explored.

\section{Acknowledgement}

This paper is supported by the Research Center at the College of Business Administration and the Deanship of Scientific Research at King Saud University, Riyadh.

\section{References}

[1] Hirsch, W.M., Dantzig, G.B., Notes on Linear Programming Part XIX, The Fixed Charge Problem, Rand Research, Memorandum No. 1383, Santa Monica, California (1954).

[2] Balinski, M.L. Fixed cost transportation problem. Naval Research Logistics Quarterly. 8, (1961)41-54.

[3] Sandrock K., A simple algorithm for solving small, fixedcharge transportation problem, Journal of Operational Research Society. 39, 5 (1988) 467-75.

[4] Rousseau, J. M., A cutting plane method for the fixed cost problem, Doctoral dissertation, Massachusetts Institute of Technology. Cambridge, MA (1973).

[5] McKeown, P. G., A vertex ranking procedure for solving the linear fixed charge problem. Operations Research. (1975)1183-1191.

[6] Palekar, U.S., M.K. Karwan, and S.Zionts, A branch-and bound method for the fixed charge transportation problem, Management Science, 36 , 9 (1990). 1092-1105.
[7] Sun, M., Aronson, J.E. Mckeown, P.G., A tabu search heuristic procedure for the fixed charge transportation problem, European Journal of Operational Research,106 (1998) 441-456.

[8] Wright, D., C. Haehling von Lanzenauer, Solving the fixed charge problem with lagrangian relaxation and cost allocation heuristics. European Journal of Operational Research, 42, (1989) 304-312.

[9] Wright, D., C. Haehling von Lanzenauer, COLE: A new heuristic approach for solving the fixed charge problem Computational results. European Journal of Operational Research, 52 (1991) 235-246.

[10] Kowalski, K and Lev, Benjamin, New Approach to Fixed charges Problems (FCP), Int. J. of Mgmt. Sci. and Engineering Mgmt. 2,1(2007) 75-80.

[11] Adlakha, V, Kowalski, K, Vemuganti, R. R. and Lev, Benjamin, More-for-less algorithm for fixed-charge transportation problems, OMEGA, The International Journal of Management Science 35,1(2007) 116-127.

[12] Adlakha, V, Kowalski, K and Lev, Benjamin, A Branching Method for the Fixed Charged Transportation Problem, OMEGA, The International Journal of Management Science 38, 5 (2010) 393-397.

[13] Lev, Benjamin and Kowalski, K., Modeling fixed-charge problems with polynomials, OMEGA, The International Journal of Management Science, 39, 6 (2011) 725-729.

[14] Kowalski, K and Lev, Benjamin, On step fixed-charge transportation problem, OMEGA, The International Journal of Management Science, 36, 5 (2008) 913-917.

[15] Hajiaghaei, M. Keshteli, S. Molla-Alizadeh-Zavardehi, R. Tavakkoli-Moghaddam, Addressing a nonlinear fixed-charge transportation problem using a spanning tree-based genetic algorithm, Computers \& Industrial Engineering, 59 (2010) 259-271. 


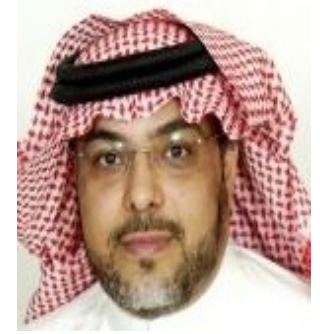

Khalid M. Altassan received the MS degree in Accounting from Oklahoma State University in 1988, other M.Sc. in Quantitative Business Analysis from Louisiana State University in 1990 and received the $\mathrm{PhD}$ degree in Engineering -Industrial and Management from EngineeringRensselaer Polytechnic Institute in 1997. His research interests are in the areas of Automated storage and Retrieval Systems, Mathematical Application in Scheduling, Quantitative analysis in Business and Economics, Operations Research Applications in Managerial Accounting.

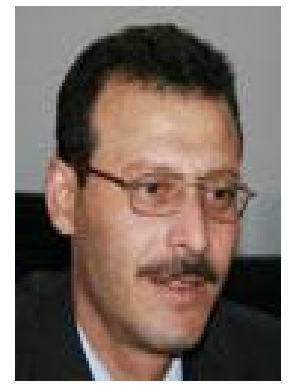

Mahmoud M. El-Sherbiny received the M.Sc. \& PhD degrees in Operations Research form Institute of Statistical Studies \& Research (ISSR), Cairo University, Egypt in 1996 and 2002 respectively. $\mathrm{He}$ is a professor in ISSR, Cairo University, Egypt . His research interests are in the areas of Computational Intelligence, Particle Swarm, Immune Systems, Quantum computing, Multiobjectives, Fuzzy Systems, Transportation and Scheduling.

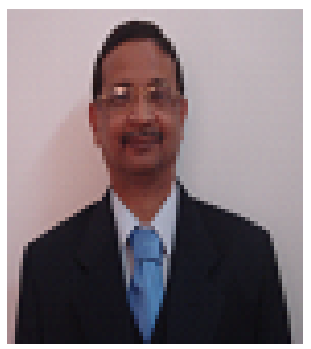

Bokkasam Sasidhar received the M.Stat. degree and P.G. Diploma in SQC \& OR from Indian Statistical Institute, Calcutta in 1977 and received the $\mathrm{PhD}$ degree in Statistics from Mangalore University in 1992. He is currently a Professor at King Saud University, Riyadh, Saudi Arabia. His research interests are in the areas of Quality Management, Quantitative analysis in Business and Operations Research Applications in Production and Management. 